\title{
Percutaneous image-guided biopsy of prostate cancer metastases yields samples suitable for genomics and personalised oncology
}

\author{
Matthew KH Hong \\ Nikhil Sapre \\ Pramit M Phal ${ }^{+}$ \\ Geoff Macintyre* \\ Xiaowen Chin \\ John S Pedersen ${ }^{\wedge \#}$ \\ Andrew Ryan ${ }^{\wedge}$ \\ Michael Kerger \\ Anthony J Costello \\ Niall M Corcoran \\ Christopher M Hovens
}

Division of Urology, Department of Surgery, University of Melbourne, Royal Melbourne Hospital and the Australian Prostate Cancer Research Centre Epworth, Richmond VIC

+ Department of Radiology, Royal Melbourne Hospital

* NICTA Victoria Research Laboratory, University of Melbourne

$\wedge$ TissuPath Specialist Pathology, Mount Waverley, VIC

\# Monash University Faculty of Medicine, Victoria, Australia

Keywords:

Prostate cancer, metastasis, guided biopsy, genomics, biorepository

Article Type: Original Research

Correspondence to:

Dr Matthew KH Hong

m.k.hong@ausdoctors.net

+61393427000 


\begin{abstract}
Personalised oncology through mutational profiling of cancers requires the procurement of fresh frozen tumour samples for genomics applications. While primary cancers are often surgically excised and therefore yield such tissue, metastases in the setting of a known cancer diagnosis are not routinely sampled prior to systemic therapy. Our study aimed to determine the suitability of extracted nucleic acids for genomics applications using distant metastatic prostate cancer samples obtained via percutaneous or surgical biopsy.
\end{abstract}

Patients with metastatic prostate cancer were recruited for image-guided biopsy of metastases. Patients undergoing surgical procedures for the complications of metastases were also recruited. Tissue samples were flash frozen and cryosectioned for histological examination. DNA and RNA were simultaneously extracted and genomic DNA hybridised onto SNP arrays for genome-wide copy number analysis.

Thirty seven samples of metastatic tissue from 7 patients with prostate cancer were obtained. Five of these underwent image-guided biopsies whilst two had therapeutic surgical procedures performed. 22 biopsy samples were obtained across the image-guided biopsy patients with $80 \%$ of samples being successfully processed for downstream analysis. Nucleic acid yield from these samples were satisfactory for genomics applications. Copy number analysis revealed a median estimated tumour purity of 53\% and all samples showed chromosomal abnormalities suggestive of malignancy.

The procurement of osseous metastatic prostate cancer from live patients, including the use of image-guided biopsy, is safe and feasible. Sufficient tissue can be obtained in a manner such that extracted nucleic acids are suitable for genomics research. 


\section{Introduction}

The last decade has seen rapid advancements in genome sequencing technology and the comprehensive survey of entire cancer genomes that has promised the advent of personalised cancer medicine[1,2]. Promising results in several cancer types have provided a rationale for the premise that driver genomic alterations within individual tumours may define patients who will clinically benefit from targeted therapies[2-4]. Focus on the clinical utility of comprehensive cancer mutation profiling is based on its potential to allow targeted therapies to be tailored for individual patients according to genetically defined tumour subtypes[2, 5]. To facilitate the successful translation of bench-top genomics to personalised cancer treatment in the clinic, a number of practical considerations have to be addressed[5].

One issue is the question of which tissue to genotype or sequence. Whilst archival samples of the primary cancer abound due to prior surgical excision, metastases are generally treated with systemic agents and are not routinely biopsied. Treatment paradigms for metastatic disease have traditionally relied on the pathological characteristics of the primary tumour. It is currently unclear if profiles of archived or prospectively stored fresh frozen primary tumour accurately represent the disease state of metastatic cancer subject to the selection pressures imposed by earlier standard systemic therapies[5]. While recent studies suggest that mutational profiling of the primary tumour might be adequate for some cancers, such as colorectal cancer where key mutations in metastases are concordant with that of the primary tumour[6,7], it is less suited for scenarios where primary cancer tissue is unavailable, driver mutations are discordant between metastasis and primary tumour, the primary cancer is highly heterogeneous, or widespread metastatic disease is treatment resistant. In addition, the drive towards biomarker-informed clinical trials will almost certainly demand the biopsy of metastatic sites initially in the nontherapeutic setting[8].

Two recent programs that have begun to address the unique challenges of personalised oncology both utilise biopsies from metastatic disease. The Michigan Oncology Sequencing Project (MI-ONCOSEQ) was a pilot study implemented to explore the practical challenges of using high-throughput sequencing in clinical oncology, and their recent report suggests that this approach is able to generate comprehensive mutational profiles to facilitate biomarker-driven clinical trials in oncology[9]. The Princess Margaret Hospital - Ontario Institute for Cancer Research-led feasibility study is similar and will include somatic mutation genotyping and targeted exome sequencing[5]. Importantly, they will examine both biopsies as well as archival samples of the primary tumours in order to determine the value of the latter.

In the last few years, various visions of personalised oncology have been forwarded with respect to the genomic platforms used, tissue format and scope of the tests employed. Earlier studies have included the use of formalinfixed archival tissue, restricted testing to just several markers or a small panel of markers, or sequenced for known prognostic mutations only[10-12]. While the trend now appears to favour genome sequencing for mutational profiling through genome sequencing, targeted or otherwise, only some emphasise the utility of whole transcriptome sequencing, which allows gene expression profiles to be integrated with mutational analysis, but necessitates the isolation of RNA from samples[9]. Such broad sequencing is better placed to match the growing need for approaches that can identify rarer genes that could have impact on clinical care[13]. Both genome and transcriptome sequencing currently require sufficient quantities of fresh frozen material with high 
quality nucleic acids as formalin can fragment DNA and introduce chemical modifications that adversely affect sequencing results[14].

Recent experience with image-guided percutaneous biopsy of breast cancer metastasis suggests that the procurement of biopsy samples suitable for such analysis is not trivial[15-18]. The inability of many biobanks to meet strict criteria for the entry of samples into large genomics studies such as the TCGA is indicative of some of the challenges facing the practical implementation of personalised medicine[19-21]. A further issue is the often limited tissue quantity available from biopsy which might well preclude its availability for formalin fixation and the subsequent histological examination that remains a cornerstone of diagnosis in surgical pathology and is required for assessment of tumour content. Cryostat sectioning could be used in its place, but the poor morphological preservation generally hampers histological evaluation and this could lead to difficulties in diagnosis and assessment of tumour purity[22].

Samples with insufficient tumour yield or content, or degraded nucleic acids, can be precluded from further analysis. However, in the clinical context where clinicians must make informed treatment decisions for every individual patient in a timely fashion, such a scenario could necessitate a repeat procedure with the additional risks and delayed treatment this would entail. Low tumour content will likely require greater depth of sequencing or, in more extreme cases, could mandate a rebiopsy for more tissue.

In this context, our study aimed to determine the suitability of extracted nucleic acids for genomics applications using distant metastatic prostate cancer samples obtained via percutaneous or surgical biopsy. We also aimed to determine the utility of copy number variation analysis on high resolution single nucleotide polymorphism (SNP) array data as a diagnostic adjunct and its potential to influence decisions regarding resampling and depth of subsequent genomic sequencing through estimation of tumour content. 


\section{Patients and Methods}

Following ethics approval, suitable prostate cancer patients were invited to undergo voluntary biopsy of their metastases. Where appropriate, this occurred in accordance with the core bioethical elements for large genomics projects[19]. Patients were primarily included where there was demonstrated evidence of metastatic disease on bone scintigraphy in the setting of biochemical recurrence after prostatectomy. Patients were also invited to donate samples if they were undergoing diagnostic or therapeutic procedures for prostate cancer metastases.

\section{Radiology and Tissue Procurement}

Patients gave informed consent and underwent a day procedure at the Department of Radiology, Royal Melbourne Hospital. Under light sedation with midazolam and fentanyl, image-guided biopsy of metastases with ultrasound or computed tomography was performed as appropriate (Fig. 1). A coaxial bone biopsy needle with an 18G internal calibre (Bonopty Bone Biopsy System, 10-1072, AprioMed AB, Sweden) was used and tissue cores were immediately placed in a $1.5 \mathrm{ml}$ microcentrifuge tube and flash frozen in liquid nitrogen. A $14 \mathrm{G}$ needle (Quick-Core Disposable Biopsy Needle, QC-14-9.0-20T, Cook, Brisbane, Australia) was used for ultrasound guided biopsies if deemed clinically appropriate. Samples were also flash frozen in liquid nitrogen and transported on dry ice. Long term storage was in liquid nitrogen vapour phase. Details of the personnel required for this procedure are presented in Table 1.

\section{Tissue Processing and Nucleic Acid Extraction}

Metastatic tissue samples were retrieved from liquid nitrogen storage and cryosectioned for histopathological assessment. Tissue samples were embedded in Optical Cutting Time compound (OCT, Sakura) at $-24^{\circ} \mathrm{C}$, and $5 \mu \mathrm{m}$ sections cut by cryotome (CM1900, Leica Microsystems, NSW, Australia). Sections were transferred to charged glass slides (Superfrost Ultra Plus, Thermo Scientific), rapidly stained with haematoxylin and eosin, and assessed in real-time by a pathologist for tumour content (Fig. 2). On confirmation of malignancy or where this was inconclusive but considered likely, the OCT-embedded tissue samples were isolated with a scalpel and placed in $700 \mu \mathrm{l}$ RLT Plus buffer for immediate homogenisation (TissueRuptor, Qiagen, CA). DNA and RNA were simultaneously extracted using the Allprep Micro Kit (Qiagen, CA) following manufacturer instructions and including on-column DNAse digestion of the RNA[23]. Genomic DNA was extracted from fresh frozen samples of whole blood with the DNeasy Blood \& Tissue Kit (Qiagen, Maryland) following manufacturer instructions. RNA quantity and quality were checked by microelectrophoresis (Agilent 2100 Bioanalyzer) whilst DNA quantity was checked by spectrophotometry (NanoDrop 1000, Thermoscientific and quality was assessed by gel electrophoresis ( $0.8 \%$ agarose gel).

\section{SNP Array}

Genomic DNA was prepared using the Infinium® HD Assay Ultra workflow (Illumina) and hybridised onto the Illumina HumanOmni2.5 BeadChip (2.5 million markers) following manufacturer's instructions. Briefly, the DNA was normalised to $200 \mathrm{ng}$ in $4 \mu \mathrm{l}$ of water, enzymatically fragmented and repurified. The library was then hybridised onto the BeadChip, which was scanned on an iScan machine (Illumina) to generate intensity values.

\section{Copy Number Analysis}

Initial data analysis was performed with GenomeStudio v2010.3 with Genotyping module 1.8.4 software (Illumina). Raw intensity values were converted to LogR ratios and B allele frequencies for each SNP. Using 
germline data from matched whole blood samples as background for minor allele frequency, copy number was called after GC correction using ASCAT (Allele-Specific Copy number Analysis of Tumours), which simultaneously estimates and adjusts for both tumour ploidy and non-aberrant cell admixture[24]. The tumour content estimate by ASCAT was compared with that provided histologically.

\section{Results}

We procured samples of metastatic tissue from 9 sites amongst 7 patients with prostate cancer (Table 2). Five patients underwent image-guided biopsy of metastases, only one of which underwent the procedure for diagnostic purposes. One patient also underwent a second procedure where two of the same metastatic sites were again sampled following androgen deprivation therapy and radiotherapy to one site. The procedures were tolerated well and no complications were encountered. The sites from which metastases were obtained included the scapula, sacrum, ilium, iliac crest, pubis, and thoracic vertebrae. We also obtained tissue from two other patients undergoing surgical procedures for metastatic disease. One patient underwent craniotomy for a large metastasis in the posterior fossa, and another underwent spinal cord decompression for a large vertebral metastasis. One patient also had locally recurrent disease sampled during transurethral resection.

Over the 5 patients who underwent image-guided biopsy, 22 cores of tissue were obtained. Ten samples representing nine metastasis sites were processed for downstream analysis (Table 3). Based on frozen section analysis, histology revealed likely cancer in 5 samples and was abnormal but inconclusive in the remainder. Two samples from a single site with recent radiotherapy treatment yielded no viable tissue and subsequent extraction of nucleic acid was not fruitful. Amongst the remaining 8 samples, median RNA yield was $1.2 \mu g$ (range 0.24$10 \mu \mathrm{g}$ ) with median RIN 9.9 (range 9.5-10, 3 samples insufficient concentration). These RNA samples have subsequently all been submitted for next-generation sequencing. Median DNA yield was 6.8 $\mu$ g (range 4.0-25 $\mu \mathrm{g}$ ) and all samples had high molecular weight DNA as checked by gel electrophoresis. Overall, only one of the nine biopsied sites of metastasis failed to yield samples suitable for downstream genomics analysis.

\section{Copy Number Analysis}

The eight samples representing metastatic lesions taken under image-guidance as well as two others from surgical procedures were hybridised onto a SNP chip and the resulting genome wide copy number information analysed. Median tumour purity in the samples was $55.5 \%$ (22\% to $89 \%)$ as estimated using ASCAT. Every sample had significant chromosomal gains or losses, consistent with malignancy and suggestive of prostate cancer in the clinical context (Fig 3). We considered this to be sufficient evidence for metastatic prostate cancer considering the confirmed diagnosis of primary prostate cancer on clinical history, the elevated PSA after prostatectomy, imaging suggestive of metastasis and abnormal histological findings on first pass. Interestingly, the suggestion of malignancy was seemingly obvious based on the highly altered B allele frequency plots, although samples with low tumour content looked benign on the BAF plots (Fig 4). We examined the two patients with multiple metastasis and where two sites were sampled in each case. While there were distinct differences between the metastases in each patient, overall the copy number landscapes appeared unique to each patient as has been previously noted (Fig 5). 


\section{Discussion}

In this study we have collected metastatic tissue samples from seven prostate cancer patients through a specific program to obtain such tissue and demonstrated that this is sufficient for multidimensional genomic profiling in each case. Our findings on SNP array data suggests that even in cases of equivocal histopathological features, genome-wide copy number profiles are likely to be sufficient to indicate malignancy. As histological analysis of cryostat sections can be difficult, we used the SNP data to estimate tumour purity, and this highlighted the low tumour content in four of our samples, for which greater depth of sequencing would likely be required for the discovery of rare mutations or robust subclone analysis[1,25].

Where possible, histological assessment showed all samples deemed to contain cancer cells to be above the 60$70 \%$ tumour cell content cut-off used in many genomic studies $[9,26]$. However, for these samples our corresponding bioinformatically derived percent tumour was about $15 \%$ lower on average, with some below the accepted cut-off. One possibility to account for this discrepancy between histological and bioinformatics assessment is the potential for admixture of the main tumour cell populations with inflammatory or stromal cells not readily accounted for on histological examination, which is the current standard for the determination of tumour cell content [5, 9]. Nevertheless, the distinct copy-number segmentation obtained from samples with as little as $22 \%$ estimated tumour cellularity (Fig 5) demonstrates that in many cases a cut-off for 60-70\% tumour cellularity is potentially too strict and genomic analyses can be performed with much lower tumour content.

One significant limitation of percutaneous image-guided biopsy in patients with metastasis is that only one to several metastatic sites can be feasibly sampled, thus potentially limiting the ability to account for the heterogeneous nature of metastatic cancer. In our particular series, four patients had solitary metastases and only two had widespread disease. Studies of anatomically distinct metastasis from rapid autopsy programs suggest a monoclonal origin in most prostate cancer metastases, maintaining a unique signature copy number pattern whilst also accumulating a variable number of separate changes[27]. This appears to have been recapitulated in our two patients where two metastatic sites were biopsied. The use of combination therapeutics based on subclonal analysis from whole genome sequencing data representative of several metastatic sites might be required to account for heterogeneity. Other limitations of our study include our bias towards bone metastases, even though diagnostic yield for bone lesions is not significantly different from soft tissue lesions[28], and the fact that it was not performed in a clinical setting with strict timelines and where clinical decisions about treatment were dependent on our results.

On the other hand, there is much experience in percutaneous image-guided biopsy and although these did not present in our series, complications such as transient lower limb paresis and haematomas occur in about $1 \%$ in large series[29]. The presence of our image-guided biopsy program also facilitated the opportunistic procurement of other metastatic tissue from diagnostic biopsies and surgical procedures for complications of metastases. Personalised medicine entails genomics analysis of cancer tissues, and so these must be obtained from therapeutic or diagnostic procedures, by biopsy or surgery. While surgery has remained the dominant modality by which solid primary cancers have been sampled for genomic sequencing, image-guided percutaneous biopsy is likely to be the vehicle by which metastatic tissue will be obtained routinely for analysis in a personalised medicine model. Surgery for metastatic disease is usually emergent and the routine availability 
of facilities for handling fresh frozen specimens may present a significant barrier. However, every metastatic tissue sample potentially represents a further opportunity to plot the evolutionary course of an individual's malignancy through genomic analysis. In our case, we were able to provide a small team with the relevant technical expertise and infrastructure to collect and process these samples fresh from surgical procedures.

The tissue and nucleic acid yields from our study appear high when compared to previous similar experiences in breast cancer metastasis patients. In their study of 34 CT-guided bone biopsies from patients with metastatic breast cancer, Hilton et al reported just $16(47 \%)$ yielding sufficient tumour cells for hormone analysis in patients with metastatic breast cancer[16]. Amir et al reported a large series of biopsies for breast cancer metastases, of which 20 were from the bone[18]. Only 15 (75\%) yielded enough tissue for immunohistochemistry. The same group reported previously a series of nine patients, where six cases yielded tumour positive samples and underwent immunohistochemical analysis[30]. In terms of nucleic acid yield, Cawthorn et al reported on 10 patients who successfully underwent CT-guided bone biopsy of metastatic breast cancer lesions[15]. Only 6 samples had sufficient RNA yield for downstream microarray analysis (50ng being the lower limit of input RNA). As numbers are small in both previous series and the present study, it is difficult to comment on why these differences exist, but it does highlight the potential for problems posed by a failure to extract sufficient nucleic acid in the clinical setting and the need for consistent protocols.

As the Bonopty needle system used in our series typically yields smaller biopsies, improvements in tissue yields are likely with the use of larger calibre biopsy sets[29].

Although personalised oncology will almost certainly entail sequencing technologies in the future, microarray technology is presently inexpensive and provides results rapidly[5]. One benefit of a high-resolution SNP array is the ability to provide data for copy number variation analysis[31]. In recent years, bioinformatics researchers have made significant progress in the techniques of copy number variation analysis with respect to cancer genomes by addressing the issues of ploidy and tumour purity[32,33]. With the ability to accurately determine percentage tumour content of samples rapidly from SNP data rather than await genome sequencing, we are now able to use this as an adjunct to aid the identification of successful sampling of tumour and to determine if the planned sequencing depth will be adequate.

In terms of research, the context in which our program is embedded, the procurement of fresh frozen paired primary and metastatic tissues from the same patients affords the opportunity to answer fundamental questions about cancer progression and metastasis[34]. Presently, metastasis samples are generally seen as a value-add rather than an essential component of biobanks[35], and the presence of more programs targeting metastatic samples has the potential to transform the research potential of many cancer biobanks that rely exclusively on surgical programs. In particular, biopsy-driven clinical trials in oncology for biomarker discovery have already begun and there is growing acceptance that this is not only feasible, but also required for the study of treatment resistance in metastatic cancer[8]. From a patient perspective, recruitment rates for these types of studies have been at least $80-90 \%$, leading to faster than expected accrual rates[18, 36].

In conclusion, percutaneous image-guided biopsy of osseous prostate cancer metastasis is safe and feasible and provides nucleic acids suitable for a range of genomics applications. Rapid analysis of genome-wide copy number alteration from SNP data can assist in the timely confirmation of malignancy and estimation of tumour 
purity prior to a commitment to further sequencing. This work represents one more step towards the practical translation of cancer genomics into routine personalised care of cancer patients.

\section{Acknowledgements}

MKHH has been supported by scholarships from the Royal Australasian College of Surgeons, the National Health and Medical Research Council, Australia, and the Melville Hughes Scholarship, Faculty of Medicine, Dentistry and Health Sciences, University of Melbourne. GM is supported by NICTA. NICTA is funded by the Australian Government as represented by the Department of Broadband, Communications and the Digital Economy and the Australian Research Council through the ICT Centre of Excellence program. We gratefully acknowledge the genomics services provided by the Australian Genome Research Facility and also the men who so kindly donated their metastatic tissues. 


\section{References}

1. Meyerson M, Gabriel S, Getz G. Advances in understanding cancer genomes through second-generation sequencing. Nat Rev Genet 2010; 11 (10): 685-96.

2. Macconaill LE, Garraway LA. Clinical implications of the cancer genome. Journal of clinical oncology : official journal of the American Society of Clinical Oncology 2010; 28 (35): 5219-28.

3. Kim ES, Herbst RS, Wistuba, II, et al. The BATTLE trial: personalizing therapy for lung cancer. Cancer discovery 2011; 1 (1): 44-53.

4. Lynch TJ, Bell DW, Sordella R, et al. Activating mutations in the epidermal growth factor receptor underlying responsiveness of non-small-cell lung cancer to gefitinib. $\mathrm{N}$ Engl $\mathrm{J}$ Med 2004; 350 (21): 2129-39.

5. Tran B, Dancey JE, Kamel-Reid S, et al. Cancer genomics: technology, discovery, and translation. Journal of clinical oncology : official journal of the American Society of Clinical Oncology 2012; 30 (6): 647-60.

6. Vakiani E, Janakiraman M, Shen R, et al. Comparative genomic analysis of primary versus metastatic colorectal carcinomas. Journal of clinical oncology : official journal of the American Society of Clinical Oncology 2012; 30 (24): 2956-62.

7. Tie J, Gibbs P, Lipton L, et al. Optimizing targeted therapeutic development: analysis of a colorectal cancer patient population with the BRAF(V600E) mutation. International journal of cancer Journal international du cancer 2011; 128 (9): 2075-84.

8. Diaz Z, Quebec Clinical Research Organization in Cancer Consortium. Feasibility and challenges of a biopsy-driven and biomarker discovery clinical trial in metastatic colorectal cancer to identify signatures of clinical resistance. ASCO Meeting Abstracts 2012; 30 (15_suppl): e14108.

9. Roychowdhury S, Iyer MK, Robinson DR, et al. Personalized oncology through integrative high-throughput sequencing: a pilot study. Science translational medicine 2011; 3 (111): $111 \mathrm{ra} 21$.

10. Thomas RK, Baker AC, Debiasi RM, et al. High-throughput oncogene mutation profiling in human cancer. Nature genetics 2007; 39 (3): 347-51.

11. MacConaill LE, Campbell CD, Kehoe SM, et al. Profiling critical cancer gene mutations in clinical tumor samples. PloS one 2009; 4 (11): e7887.

12. Dias-Santagata D, Akhavanfard S, David SS, et al. Rapid targeted mutational analysis of human tumours: a clinical platform to guide personalized cancer medicine. EMBO molecular medicine 2010; 2 (5): 146-58.

13. Corless CL. Medicine. Personalized cancer diagnostics. Science 2011; 334 (6060): 1217-8.

14. Garraway LA. Concordance and discordance in tumor genomic profiling. Journal of clinical oncology : official journal of the American Society of Clinical Oncology 2012; 30 (24): 2937-9.

15. Cawthorn TR, Amir E, Broom R, et al. Mechanisms and pathways of bone metastasis: challenges and pitfalls of performing molecular research on patient samples. Clinical \& experimental metastasis 2009; 26 (8): 935-43.

16. Hilton JF, Amir E, Hopkins S, et al. Acquisition of metastatic tissue from patients with bone metastases from breast cancer. Breast cancer research and treatment 2011; 129 (3): 761 5.

17. Amir E, Clemons M, Purdie CA, et al. Tissue confirmation of disease recurrence in breast cancer patients: pooled analysis of multi-centre, multi-disciplinary prospective studies. Cancer treatment reviews 2012; 38 (6): 708-14.

18. Amir E, Miller N, Geddie W, et al. Prospective study evaluating the impact of tissue confirmation of metastatic disease in patients with breast cancer. Journal of clinical oncology : official journal of the American Society of Clinical Oncology 2012; 30 (6): 587-92. 
19. Hudson TJ, Anderson W, Artez A, et al. International network of cancer genome projects. Nature 2010; 464 (7291): 993-8.

20. Blow N. Biobanking: freezer burn. Nature Methods 2009; 6 (2): 173-8.

21. Hewitt RE. Biobanking: the foundation of personalized medicine. Current opinion in oncology 2011; 23 (1): 112-9.

22. Alers JC, Krijtenburg PJ, Vissers KJ, et al. Effect of bone decalcification procedures on DNA in situ hybridization and comparative genomic hybridization. EDTA is highly preferable to a routinely used acid decalcifier. The journal of histochemistry and cytochemistry : official journal of the Histochemistry Society 1999; 47 (5): 703-10.

23. Xu C, Houck JR, Fan W, et al. Simultaneous isolation of DNA and RNA from the same cell population obtained by laser capture microdissection for genome and transcriptome profiling. J Mol Diagn 2008; 10 (2): 129-34.

24. Van Loo P, Nordgard SH, Lingjaerde OC, et al. Allele-specific copy number analysis of tumors. Proceedings of the National Academy of Sciences of the United States of America 2010; 107 (39): 16910-5.

25. Nik-Zainal S, Van Loo P, Wedge DC, et al. The life history of 21 breast cancers. Cell 2012; 149 (5): 994-1007.

26. Taylor BS, Schultz N, Hieronymus H, et al. Integrative genomic profiling of human prostate cancer. Cancer cell 2010; 18 (1): 11-22.

27. Liu W, Laitinen S, Khan S, et al. Copy number analysis indicates monoclonal origin of lethal metastatic prostate cancer. Nat Med 2009; 15 (5): 559-65.

28. Wu JS, Goldsmith JD, Horwich PJ, et al. Bone and soft-tissue lesions: what factors affect diagnostic yield of image-guided core-needle biopsy? Radiology 2008; 248 (3): 962-70.

29. Rimondi E, Rossi G, Bartalena T, et al. Percutaneous CT-guided biopsy of the musculoskeletal system: results of 2027 cases. European journal of radiology 2011; 77 (1): $34-42$.

30. Amir E, Ooi WS, Simmons C, et al. Discordance between receptor status in primary and metastatic breast cancer: an exploratory study of bone and bone marrow biopsies. Clin Oncol (R Coll Radiol) 2008; 20 (10): 763-8.

31. Yau C, Holmes CC. CNV discovery using SNP genotyping arrays. Cytogenetic and genome research 2008; 123 (1-4): 307-12.

32. Li A, Liu Z, Lezon-Geyda K, et al. GPHMM: an integrated hidden Markov model for identification of copy number alteration and loss of heterozygosity in complex tumor samples using whole genome SNP arrays. Nucleic acids research 2011; 39 (12): 4928-41.

33. Wineinger NE, Kennedy RE, Erickson SW, et al. Statistical issues in the analysis of DNA Copy Number Variations. International journal of computational biology and drug design 2008; 1 (4): 368-95.

34. Stoecklein NH, Klein CA. Genetic disparity between primary tumours, disseminated tumour cells, and manifest metastasis. International journal of cancer Journal international du cancer 2010; 126 (3): 589-98.

35. Dhir R. Prostate cancer biobanking. Curr Opin Urol 2008; 18 (3): 309-14.

36. Simmons C, Miller N, Geddie W, et al. Does confirmatory tumor biopsy alter the management of breast cancer patients with distant metastases? Annals of oncology : official journal of the European Society for Medical Oncology / ESMO 2009; 20 (9): 1499-504. 


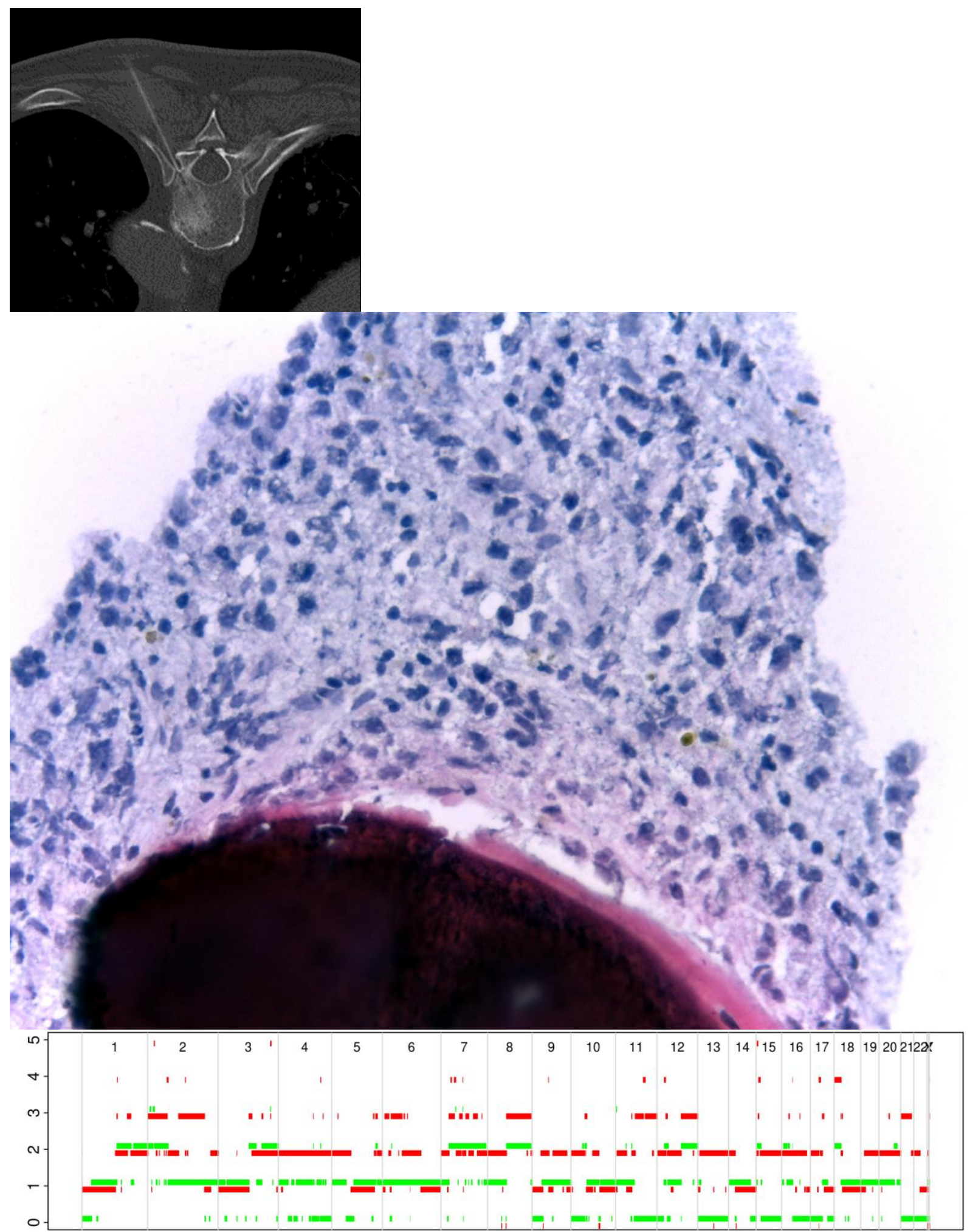



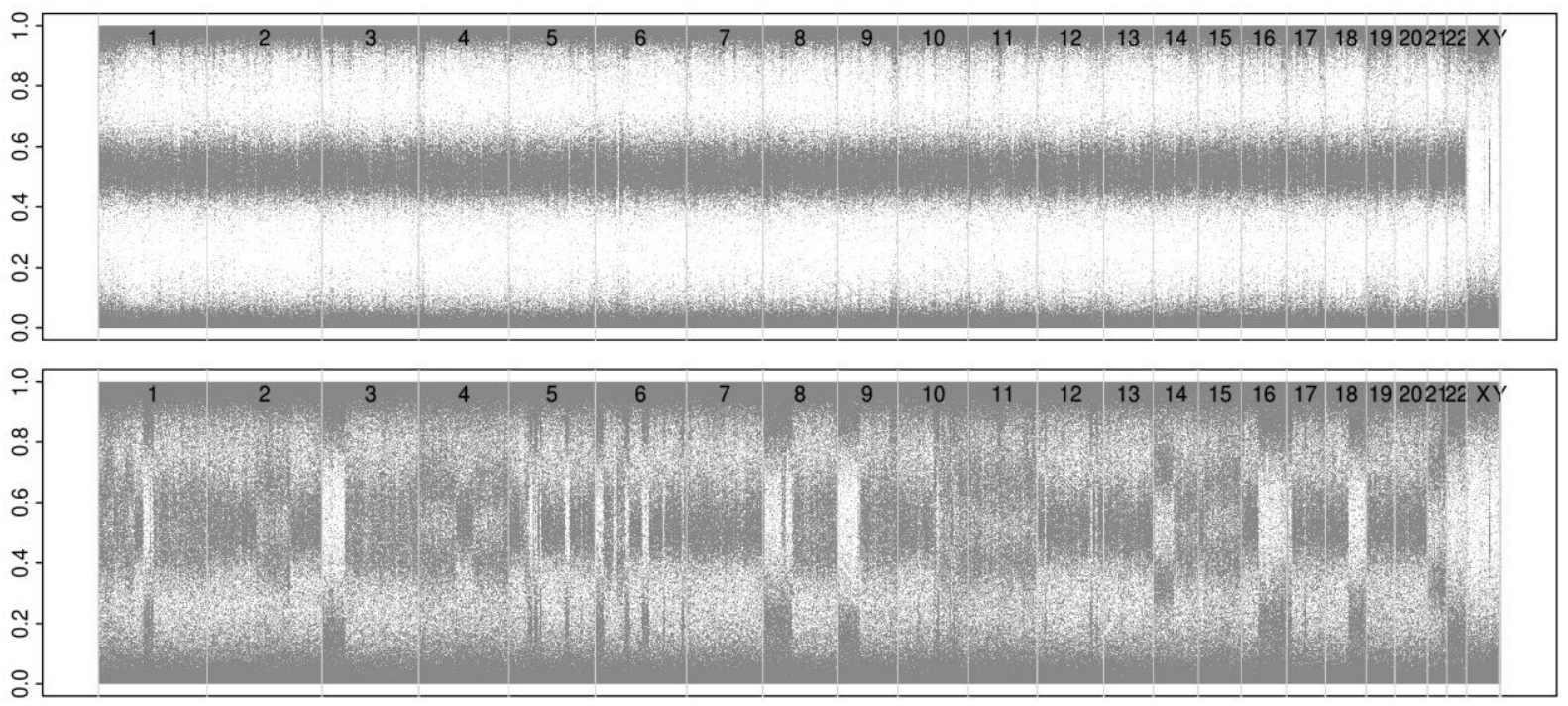

Ploidy: 2.21, aberrant cell fraction: $49 \%$, goodness of fit: $98.5 \%$

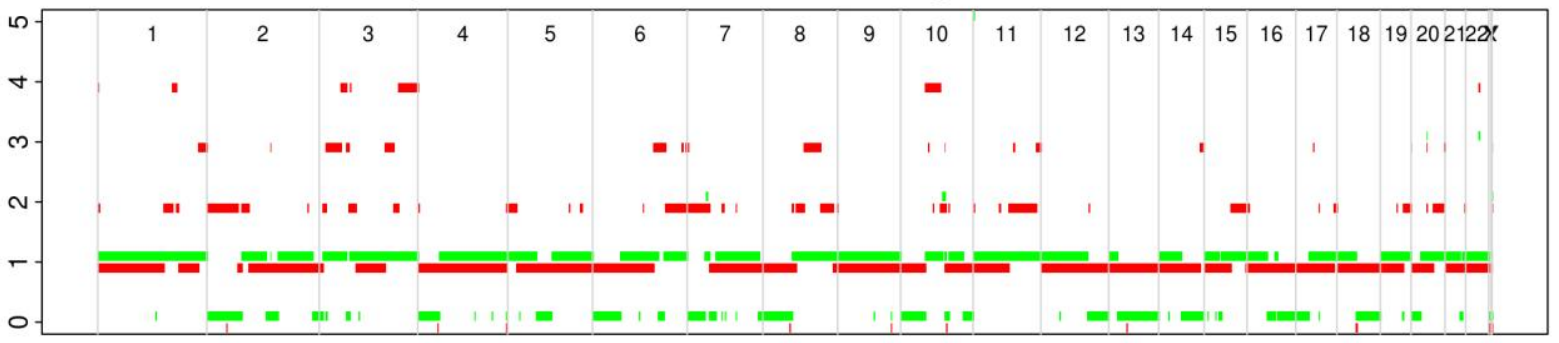

Ploidy: 2.20 , aberrant cell fraction: $46 \%$, goodness of fit: $96.5 \%$

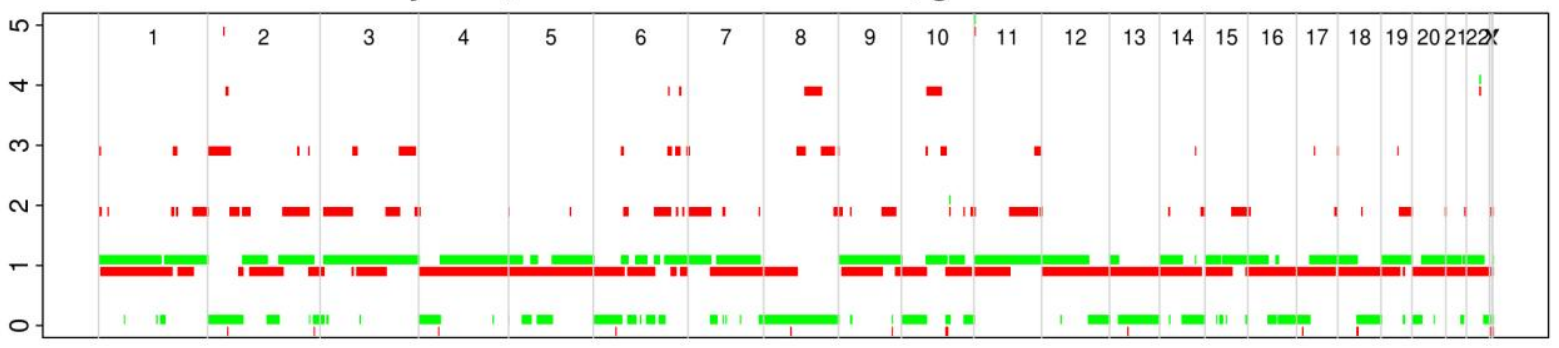

Ploidy: 3.03 , aberrant cell fraction: $82 \%$, goodness of fit: $99.2 \%$

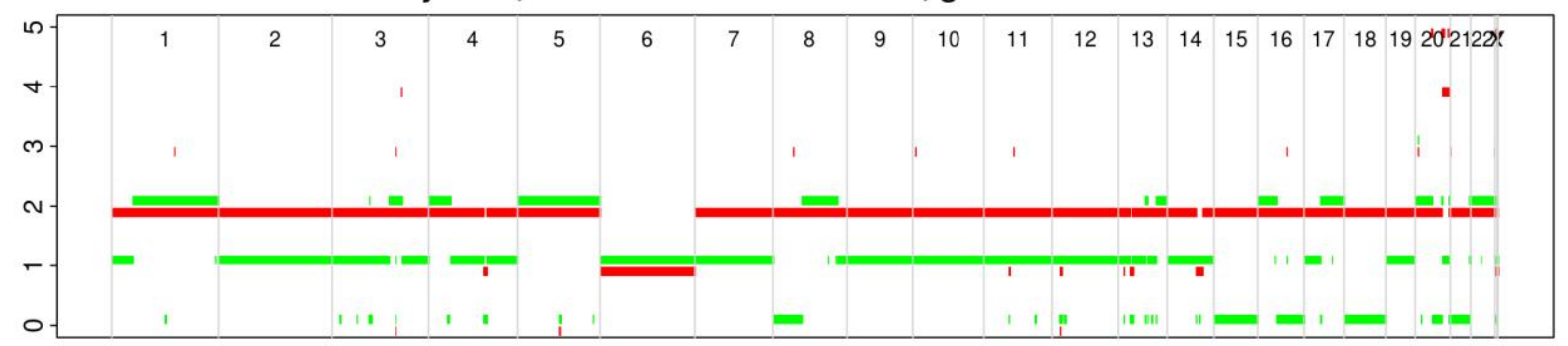

Ploidy: 3.30 , aberrant cell fraction: $22 \%$, goodness of fit: $95.7 \%$

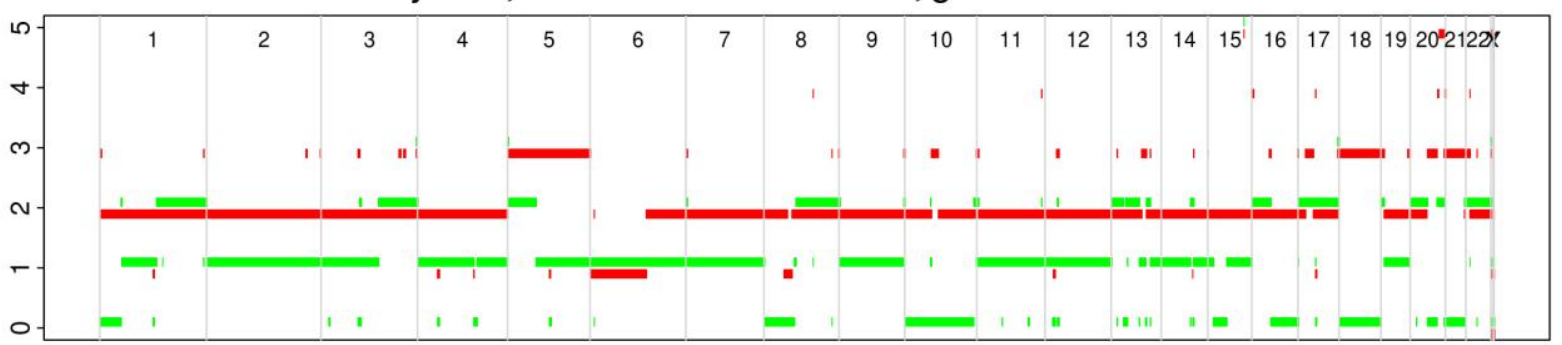

Table 1. Personnel required for sampling metastatic bone lesions under radiological guidance for research purposes. 


\begin{tabular}{|ll|}
\hline Component & Personnel \\
\hline Radiology & Radiographers \\
& Radiologist \\
\hline Laboratory & Technician \\
& Coordinator/Patient Liaison \\
\hline Clinical (Recruitment) & Clinician \\
\hline
\end{tabular}

Table 2: Details of metastatic tissue procurement from prostate cancer patients

\begin{tabular}{|llllll|}
\hline Pt & Modality & Metastases & Sites Sampled & Samples & Cancer Confirmed Histologically \\
\hline 1 & US & 1 & Scapula & 2 & Yes \\
\hline $2^{1}$ & CT & Many & Sacrum & 2 and 2 & No \\
& & & Iliac crest & 2 and 4 & Yes \\
\hline 3 & CT & 6 & Pubis & 2 & No \\
& & & Pelvic wall & 2 & Yes \\
\hline 4 & CT & 1 & Ilium & 4 & No \\
\hline 5 & CT & 1 & Vertebral & 2 & Yes \\
\hline 6 & Surgery $^{2}$ & 1 & Vertebral & 2 & Yes \\
\hline 7 & Surgery $^{3}$ & Many & Brain & 15 & Yes \\
\hline
\end{tabular}

1. Local recurrence also banked, hormone naïve and castrate resistant metastases serially harvested

2. Spinal decompression

3. Craniotomy

Table 3. Samples of metastatic prostate cancer with tumour purity and nucleic acid yields

\begin{tabular}{|l|l|l|l|r|r|r|}
\hline Sample & Site & Histology & $\begin{array}{l}\text { Tumour } \\
\boldsymbol{\%}\end{array}$ & $\begin{array}{l}\text { RNA } \\
(\boldsymbol{\mu g})\end{array}$ & $\begin{array}{l}\text { DNA } \\
(\boldsymbol{\mu g})\end{array}$ & $\begin{array}{l}\text { ASCAT } \\
\text { Fraction }\end{array}$ \\
\hline $067 \mathrm{aM} 2$ & Vertebra & Cancer & $99 \%$ & 10 & 25 & $69 \%$ \\
\hline $299 \mathrm{aM}$ & Scapula & Cancer & $80 \%$ & 5.92 & 10.9 & $75 \%$ \\
\hline $498 \mathrm{fTM} 2$ & Ilium & Cancer & $80 \%$ & 1.76 & 6.1 & $53 \%$ \\
\hline $498 \mathrm{cM}$ & Ilium & Uncertain & & 1.52 & 7.4 & $22 \%$ \\
\hline $498 \mathrm{aM}$ & Sacrum & Cancer & $99 \%$ & 0.32 & 10.3 & $82 \%$ \\
\hline $80001 \mathrm{bM}$ & Pubis & Uncertain & & 0.44 & 1.7 & $49 \%$ \\
\hline $80001 \mathrm{gM}$ & Pelvic Wall & Cancer & $\mathbf{6 0 \%}$ & 0.24 & 4.5 & $46 \%$ \\
\hline $80177 \mathrm{cM}$ & Iliac Crest & Uncertain & & 0.88 & 4.0 & $23 \%$ \\
\hline & & & & & & \\
\hline $80068 \mathrm{aMet}$ & Vertebra & Uncertain & $70 \%$ & 1.08 & 11.9 & $58 \%$ \\
\hline $80002 \mathrm{aM}$ & Cerebellum & Cancer & $90 \%$ & 6.72 & 151 & $89 \%$ \\
\hline
\end{tabular}

